\title{
Distortion Analysis in OQAM/FBMC-based OFDMA
}

\author{
David Gregoratti and Xavier Mestre \\ Centre Tecnològic de Telecomunicacions de Catalunya (CTTC) \\ Parc Mediterrani de la Tecnologia - 08860 - Castelldefels - Barcelona (Spain) \\ email: first.last@cttc.es
}

\begin{abstract}
The paper considers an OFDMA scheme based on OQAM/FBMC and evaluates the interaction of the different users to one another under strong frequency selectivity or timing synchronization errors between users. More specifically, due to imperfect equalization, each user contributes to a distortion component in the received signal. Such a distortion affects both its assigned subcarriers as well as others'. The distortion analytical expression shows the relationship between the prototype pulses and the number of affected out-band subcarriers. Realistic study cases show that OQAM/FBMC is a suitable solution for OFDMA schemes, since distortion can be maintained at tolerable levels.
\end{abstract}

\section{INTRODUCTION}

In a multiple access channel, for example the uplink communication between users and base station in a cellular network, a number of transmitters share the available resources (bandwidth, time, ...) in order to convey their message to a common receiver. The idea behind Orthogonal Frequency Division Multiple Access (OFDMA) is to divide the system bandwidth into a set of orthogonal subcarriers that are dynamically assigned to the different users. Since users transmit on orthogonal channels, they do not interfere with one another. Smart allocation policies that take into account channel quality and throughput requirements can thus be designed to maximize the transmission sum-rate. Furthermore, if the number of subcarriers is high enough, the channel frequency response can be considered flat over each subband [1], [2].

In practical systems, however, the orthogonality among subcarriers is not perfect and multiple-access interference cannot be completely avoided. For example, the classic OFDMA scheme based on Cyclic Prefix (CP-OFDMA) is very sensitive to timing errors and carrier frequency offsets. Therefore, sophisticated algorithms should be provided to synchronize all users accessing the channel. Typically, this is not enough and further processing is needed to cancel the residual interference. The computational complexity is thus an issue in these schemes [2].

An interesting alternative is offered by Filterbank Multiple Carrier (FBMC) modulations. Indeed, when the prototype pulses are ideal (i.e., they have perfect reconstruction properties, see [3]) and the channel is perfectly equalized, the low side-lobe filters of FBMC guarantee a greater robustness to

This work was partially supported by the Catalan Government under grant 2014 SGR 1567 and by the European Commission under project 7FP ICT 318362 . timing errors and frequency offsets than the CP-OFDMA approach. This means that there is hopefully no need for complex synchronization algorithms or interference cancellation techniques to achieve a reasonable level of orthogonality among subcarriers even when the prototype pulse is suboptimal and/or the channel equalization is only approximate [4].

This paper characterizes an FBMC-based OFDMA channel with a single tap per subcarrier equalizer. All channels are assumed to be highly frequency selective and/or there is no timing synchronization between users. Following the approach in [5] we derive an accurate approximation of the distortion caused by each user. Note that, as compared to [5], the multiuser case presents some specific difficulties that arise from partitioning the subcarriers into user sets. Indeed, important properties of the involved operations (e.g., the Discrete Fourier Transform-DFT) do not hold if applied to portions-and not to the totality-of the spectrum.

The distortion analysis presented hereafter is thus a powerful tool to estimate the performance of an OFDMA channel based on FBMC. Specifically, we will show that the distortion generated by each user affects not only the users' subcarriers (in-band distortion) but also leaks into the band assigned to the adjacent users (out-band distortion). Interestingly, channel frequency responses and timing synchronization errors play a role only in the magnitude of the distortion. Conversely, the number of subcarriers where the out-band distortion is significant depends exclusively on the filterbank prototype pulses. Thus, if inter-user interference is to be avoided by placing guard-bands between them, the corresponding number of inactive subcarriers can be computed without any knowledge about the users' channels.

\section{Channel Model}

We consider a multiple access channel based on Offset Quadrature Amplitude Modulation FBMC (OQAM/FBMC): the total number $2 M$ of available subcarriers is partitioned into $K$ disjoint subsets $\mathcal{I}_{k}$ that are assigned to the corresponding users $k=1, \ldots, K$. Let $\mathbf{A}_{k}=\mathbf{B}_{k}+j \mathbf{C}_{k}$ be the $2 M \times N_{s}$ matrix gathering a block of $N_{s}$ multicarrier complex symbols for user $k$. For $m \in \mathcal{I}_{k}$, symbols $\left[\mathbf{B}_{k}\right]_{m, n}$ and $\left[\mathbf{C}_{k}\right]_{m, n}$ are modeled as real-valued, independent (across $k, m$ and $n$ ) and identically distributed random variables, for all $n=1, \ldots, N_{s}$. Conversely, $\left[\mathbf{A}_{k}\right]_{m, n}=0$ for all $m \notin \mathcal{I}_{k}$, that is for all subcarriers not assigned to user $k$. 
Assume that symbols in $\mathbf{A}_{k}$ are transmitted over a perfect channel (noiseless and memoryless) with an OQAM/FBMC modulation. Also, denote by $p_{N}[n]$ and $q_{N}[n]$ the real-valued prototype pulses at the transmitter and receiver side, respectively. Both pulses have length $N=2 M \kappa$, with integer overlapping factor $\kappa$. Then, the output of the demodulator at the receiver side (see Fig. 1) is given by

$\mathbf{Y}_{k}\left(p_{N}, q_{N}\right)=\mathbf{Y}_{k}^{\text {even }}\left(p_{N}, q_{N}\right) \otimes[1,0]+\mathbf{Y}_{k}^{\text {odd }}\left(p_{N}, q_{N}\right) \otimes[0,1]$,

where $\otimes$ stands for the Kronecker product and

$$
\begin{array}{r}
\mathbf{Y}_{k}^{\text {odd }}\left(p_{N}, q_{N}\right)=2 \boldsymbol{\Phi}_{2 M}^{H}\left(\left[\mathbf{F}_{2 M} \boldsymbol{\Phi}^{*} \mathbf{B}_{k}, \mathbf{0}, \mathbf{0}\right] \circledast \mathcal{R}\left(p_{N}, q_{N}\right)\right) \\
+2 \boldsymbol{\Phi} \mathbf{F}_{2 M}^{H}\left(\left[\begin{array}{l}
\mathbf{0}, j \mathbf{G}_{2} \boldsymbol{\Phi}^{*} \mathbf{C}_{k}, \mathbf{0} \\
j \mathbf{G}_{1} \boldsymbol{\Phi}^{*} \mathbf{C}_{k}, \mathbf{0}, \mathbf{0}
\end{array}\right] \circledast \mathcal{S}\left(p_{N}, q_{N}\right)\right),
\end{array}
$$

$$
\begin{aligned}
\mathbf{Y}_{k}^{\text {even }}\left(p_{N}, q_{N}\right)= & 2 \boldsymbol{\Phi} \mathbf{F}_{2 M}^{H}\left(\left[\mathbf{0}, j \mathbf{F}_{2 M} \boldsymbol{\Phi}^{*} \mathbf{C}_{k}, \mathbf{0}\right] \circledast \mathcal{R}\left(p_{N}, q_{N}\right)\right) \\
& +2 \boldsymbol{\Phi} \mathbf{F}_{2 M}^{H}\left(\left[\begin{array}{l}
\mathbf{0}, \mathbf{G}_{2} \boldsymbol{\Phi}^{*} \mathbf{B}_{k}, \mathbf{0} \\
\mathbf{G}_{1} \boldsymbol{\Phi}^{*} \mathbf{B}_{k}, \mathbf{0}, \mathbf{0}
\end{array}\right] \circledast \mathcal{S}\left(p_{N}, q_{N}\right)\right) .
\end{aligned}
$$

In the definitions above we have introduced the diagonal matrix $\boldsymbol{\Phi}=\operatorname{diag}_{m=1, \ldots, 2 M}\left\{e^{-j \pi \frac{M+1}{2 M}(m-1)}\right\}$ and the $2 M \times 2 M$ Fourier matrix $\mathbf{F}_{2 M}$ with entries $\left[\mathbf{F}_{2 M}\right]_{m, n}=$ $(2 M)^{-1 / 2} e^{j \frac{2 \pi}{2 M}(m-1)(n-1)}$. The upper (respectively, lower) $M$ rows of $\mathbf{F}_{2 M}$ are denoted $\mathbf{G}_{1}$ (respectively, $\mathbf{G}_{2}$ ), i.e. $\mathbf{F}_{2 M}=\left[\mathbf{G}_{1}^{T} \mathbf{G}_{2}^{T}\right]^{T}$. Furthermore, using the row-wise convolution operator $\circledast$, the matrices $\mathcal{R}\left(p_{N}, q_{N}\right)$ and $\mathcal{S}\left(p_{N}, q_{N}\right)$ are built according to

$$
\begin{aligned}
& \mathcal{R}\left(p_{N}, q_{N}\right)=\left[\begin{array}{l}
\mathbf{P}_{1} \circledast \mathbf{J}_{M} \mathbf{Q}_{2} \\
\mathbf{P}_{2} \circledast \mathbf{J}_{M} \mathbf{Q}_{1}
\end{array}\right], \\
& \mathcal{S}\left(p_{N}, q_{N}\right)=\left[\begin{array}{l}
\mathbf{P}_{2} \circledast \mathbf{J}_{M} \mathbf{Q}_{2} \\
\mathbf{P}_{1} \circledast \mathbf{J}_{M} \mathbf{Q}_{1}
\end{array}\right],
\end{aligned}
$$

where $\mathbf{J}_{M}$ is the $M \times M$ anti-identity matrix (all entries are zero except for those on the main anti-diagonal, which are set to one). Matrices $\mathbf{P}_{1}$ and $\mathbf{P}_{2}$ are the top half and the bottom half, respectively, of the $2 M \times \kappa$ matrix

$$
\mathbf{P}=\left[\begin{array}{c}
\mathbf{P}_{1} \\
\mathbf{P}_{2}
\end{array}\right]=\left[\begin{array}{ccc}
p_{N}[1] & \cdots & p_{N}[2 M(\kappa-1)+1] \\
\vdots & & \vdots \\
p_{N}[2 M] & \cdots & p_{N}[2 M \kappa]
\end{array}\right] .
$$

Note that the $m$-th row of $\mathbf{P}$ is the $m$-th Type-I polyphase component of the prototype pulse $p_{N}[n]$. Matrix $\mathbf{Q}=\left[\mathbf{Q}_{1}^{T} \mathbf{Q}_{2}^{T}\right]^{T}$ is built similarly from the receiver pulse $q_{N}[n]$.

It is well-known [3], [5], [6] that the transmitted symbols in $\mathbf{A}_{k}$ can be perfectly reconstructed from the demodulated symbols in $\mathbf{Y}_{k}\left(p_{N}, q_{N}\right)$ by taking

$$
\begin{aligned}
{\left[\mathbf{A}_{k}\right]_{m, n}=\operatorname{Re}\left[\mathbf{Y}_{k}^{\text {odd }}\left(p_{N}, q_{N}\right)\right]_{m, n+\kappa-1} } & \\
& +j \operatorname{Im}\left[\mathbf{Y}_{k}^{\text {even }}\left(p_{N}, q_{N}\right)\right]_{m, n+\kappa} .
\end{aligned}
$$

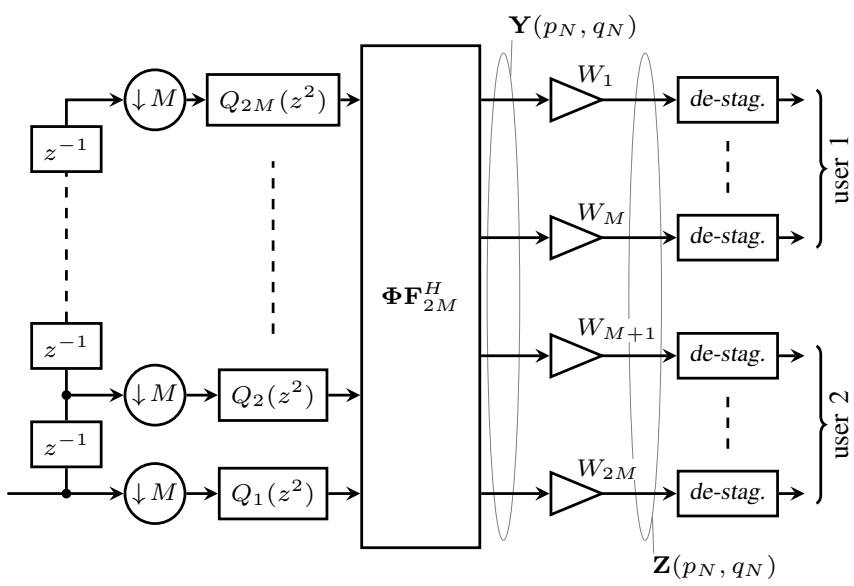

Fig. 1. Receiver block diagram assuming that subcarriers are equally split between two users. Blocks labeled "de-stag." represent the de-staggering operation (see, e.g., [5]). The coefficients of the polyphase filters $Q_{m}\left(z^{-2}\right)=$ $\sum_{n=1}^{\kappa} q_{N}[m+2(n-1) M] z^{-2(n-1)}$ are given by the $m$-th row of matrix $\mathbf{Q}$.

This property holds, however, only if the matrices $\mathcal{R}\left(p_{N}, q_{N}\right)$ and $\mathcal{S}\left(p_{N}, q_{N}\right)$ satisfy the reconstruction constraints

$$
\mathbf{U}^{+} \mathcal{R}\left(p_{N}, q_{N}\right)=\mathbb{I} \quad \mathbf{U}^{-} \mathcal{S}\left(p_{N}, q_{N}\right)=\mathbf{0}_{2 M \times(2 \kappa-1)}
$$

where we have defined $\mathbf{U}^{ \pm}=\left(\mathbf{I}_{2} \otimes\left(\mathbf{I}_{M} \pm \mathbf{J}_{M}\right)\right)$ and $\mathbb{I}=\left[\mathbf{0}_{2 M \times(\kappa-1)}, \mathbf{1}, \mathbf{0}_{2 M \times(\kappa-1)}\right]$, with $\mathbf{I}_{X}$ the $X \times X$ identity matrix and 1 a column vector of $2 M$ ones.

In practical systems, where channels are frequency selective and/or there exist timing synchronization errors, (1) does not serve anymore as a model for the demodulated symbols. Note that a delay is equivalent to a phase shift in the end-to-end channel frequency response and, thus, the two impairments are equivalent and can be treated as one. Keeping these considerations in mind, a more useful approximation for the receiver depicted in Fig. 1 is [5]

$$
\begin{aligned}
\mathbf{Z}_{k}^{(*)}\left(p_{N}, q_{N}\right) & =\mathbf{W} \boldsymbol{\Lambda}_{H_{k}} \mathbf{Y}_{k}^{(*)}\left(p_{N}, q_{N}\right) \\
- & \frac{j}{2 M} \mathbf{W} \boldsymbol{\Lambda}_{H_{k}^{(1)}} \mathbf{Y}_{k}^{(*)}\left(p_{N}, q_{N}^{(1)}\right) \\
- & \frac{1}{8 M^{2}} \mathbf{W} \boldsymbol{\Lambda}_{H_{k}^{(2)}} \mathbf{Y}_{k}^{(*)}\left(p_{N}, q_{N}^{(2)}\right)+\mathbf{o}\left(M^{-2}\right)
\end{aligned}
$$

where $(*) \in\{$ odd, even $\}$ and where

- the diagonal matrix $\mathbf{W}$ collects the $2 M$ coefficients of a channel equalizer with a single tap per subcarrier;

- the $m$-th entry of the diagonal matrix $\boldsymbol{\Lambda}_{H_{k}^{(r)}}$ is the term corresponding to the $m$-th subcarrier of the $r$-th derivative of the channel frequency response $H_{k}(\omega)$.

- the symbols in $\mathbf{Y}_{k}^{(*)}\left(p_{N}, q_{N}^{(r)}\right)$ are built as in (2) after substituting the receiver prototype pulse $q_{N}$ with its $r$-th derivative $q_{N}^{(r)}$;

- $\mathbf{o}\left(M^{-2}\right)$ represents a matrix of appropriate size whose entries decay faster than $M^{-2}$ as $M \rightarrow+\infty$.

Approximation (5) holds for large-enough $M$ and when the prototype pulses $p_{N}$ and $q_{N}$ satisfy some mild assumptions that are specified in [5]. An example is depicted in Fig. 2.

Note that, for the sake of simplicity, we limit the approximation in (5) to the second order since further terms do not bring 


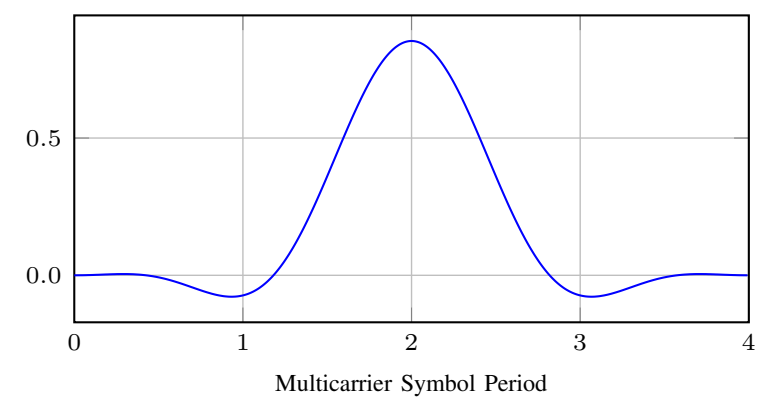

Fig. 2. Prototype pulse defined by the PHYDYAS EU-funded project [7] with overlapping factor $\kappa=4$ : it is a good approximation of a prototype pulse satisfying the required assumptions.

a significant contribution to the analysis below. However, the approximation can be straightforwardly extended to any larger order.

\section{Distortion AnAlysis}

\section{A. Preliminary Considerations}

In this section we will evaluate the distortion that is introduced when the transmitted symbols are estimated from the demodulated symbols in $\mathbf{Z}_{k}\left(p_{N}, q_{N}\right)$. More specifically, following (4) and for any $m \in \mathcal{I}_{k}$ and any $n=1, \ldots, N_{s}$, the chosen estimate for symbol $\left[\mathbf{A}_{k}\right]_{m, n}$ is

$$
\begin{aligned}
\tilde{a}_{m, n}=\sum_{s=1}^{K} \mathbb{R e}\left[\mathbf{Z}_{s}^{\text {odd }}\left(p_{N}, q_{N}\right)\right]_{m, n+\kappa-1} \\
\\
\quad+j \sum_{s=1}^{K} \mathbb{I m}\left[\mathbf{Z}_{s}^{\text {even }}\left(p_{N}, q_{N}\right)\right]_{m, n+\kappa}
\end{aligned}
$$

since the contributions of the different users combine linearly at the receiver side. Now, recalling that $\mathbf{A}_{k}=\mathbf{B}_{k}+j \mathbf{C}_{k}$, with $\left[\mathbf{B}_{k}\right]_{m, n}$ and $\left[\mathbf{C}_{k}\right]_{m, n}$ i.i.d., zero mean for all $m \in \mathcal{I}_{k}$ and $\left[\mathbf{B}_{k}\right]_{m, n}=\left[\mathbf{C}_{k}\right]_{m, n}=0$ for all $m \notin \mathcal{I}_{k}$, the distortion power at subcarrier $m$ can be written as

$$
\begin{aligned}
P_{e}(m) & =\mathbb{E}\left[\left|\tilde{a}_{m, n}-\left[\mathbf{A}_{k}\right]_{m, n}\right|^{2}\right] \\
& =2 \sum_{s=1}^{K} \mathbb{E}\left[\left|\mathbb{R e}\left[\mathbf{Z}_{s}^{\text {odd }}\left(p_{N}, q_{N}\right)\right]_{m, n+\kappa-1}-\left[\mathbf{B}_{s}\right]_{m, n}\right|^{2}\right] .
\end{aligned}
$$

Thus, we only need to compute the contribution of each user to the distortion at subcarrier $m$, namely

$$
P_{e, k}(m)=2 \mathbb{E}\left[\left|\mathbb{R e}\left[z_{k, m}^{\text {odd }}\right]-b_{k, m}\right|^{2}\right],
$$

where we wrote $z_{k, m}^{\text {odd }}=\left[\mathbf{Z}_{k}^{\text {odd }}\left(p_{N}, q_{N}\right)\right]_{m, n+\kappa-1}$ and $b_{k, m}=$ $\left[\mathbf{B}_{k}\right]_{m, n}$ to simplify notation. Note that there are no assumptions about whether $m \in \mathcal{I}_{k}$ (in-band distortion) or $m \notin \mathcal{I}_{k}$ (out-band distortion). Consequently, the expression derived hereafter is common to both cases and holds for both $m \in \mathcal{I}_{k}$ and $m \notin \mathcal{I}_{k}$.

\section{B. Distortion Expression}

Let $y_{k, m}^{\text {odd }}\left(p_{N}, q_{N}^{(r)}\right)=\left[\mathbf{Y}_{k}^{\text {odd }}\left(p_{N}, q_{N}^{(r)}\right)\right]_{m, n+\kappa-1}$. Then, using (5), we can write

$$
\begin{aligned}
\mathbb{R e}\left[z_{k, m}^{\text {odd }}\right]- & b_{k, m}=\mathbb{R e}\left[W_{m} H_{k, m}\left(y_{k, m}^{\text {odd }}\left(p_{N}, q_{N}\right)-b_{k, m}\right)\right. \\
& -\frac{j}{2 M} W_{m} H_{k, m}^{(1)} y_{k, m}^{\text {odd }}\left(p_{N}, q_{N}^{(1)}\right) \\
& \left.-\frac{1}{8 M^{2}} W_{m} H_{k, m}^{(2)} y_{k, m}^{\text {odd }}\left(p_{N}, q_{N}^{(2)}\right)\right]+o\left(M^{-2}\right),
\end{aligned}
$$

where $W_{m}$ and $H_{k, m}^{(r)}$ are the $m$-th diagonal elements of the matrices $\mathbf{W}$ and $\boldsymbol{\Lambda}_{H_{k}^{(r)}}$, respectively.

Consider now two prototype pulses $p_{N}[n]$ and $q_{N}[n]$ that satisfy the required assumptions together with the reconstruction constraints (3). Furthermore, assume that they are symmetric, meaning that, e.g., $p_{N}[n]=p_{N}[2 M \kappa-n+1]$. Then, after some algebra (the full proof will be given in the journal version of this paper, in preparation), the following result can be derived:

$$
\begin{array}{r}
\mathbb{E}\left[\left|\operatorname{Re}\left[z_{k}^{\text {odd }}\left(p_{N}, q_{N}\right)\right]-b_{k, m}\right|^{2}\right]=\eta_{0,0}+\frac{2}{2 M} \eta_{0,1} \\
+\frac{1}{4 M^{2}} \eta_{0,2}+\frac{1}{4 M^{2}} \eta_{1,1}+o\left(M^{-2}\right),
\end{array}
$$

where the $\eta_{x, y}$ terms are given by equations (7) at the top of the next page. The new matrices used in the definition of the terms $\eta_{x, y}$ are:

$$
\begin{aligned}
& \mathcal{F}_{k}(m)=\mathbf{F}_{2 M}^{H} \mathbb{E}\left[\mathbf{b}_{k} \mathbf{b}_{k}^{H}\right] \mathbf{F}_{2 M} \odot \mathbf{f}(m) \mathbf{f}^{H}(m), \\
& \chi_{\left(p, q^{\prime}, p, q^{\prime}\right)}^{\mathcal{R}}=\mathcal{R}\left(p_{N}, q_{N}^{\prime}\right) \mathcal{R}^{T}\left(p_{N}, q_{N}^{\prime}\right), \\
& \chi_{(p, q, p, q)}^{\mathcal{R}-\frac{1}{2} \mathbb{I}}=\left(\mathcal{R}\left(p_{N}, q_{N}\right)-\frac{1}{2} \mathbb{I}\right)\left(\mathcal{R}\left(p_{N}, q_{N}\right)-\frac{1}{2} \mathbb{I}\right)^{T}, \\
& \chi_{\left(p, q, p, q^{(r)}\right)}^{\mathcal{R}-\frac{1}{2} \mathbb{I}, \mathcal{R}}=\left(\mathcal{R}\left(p_{N}, q_{N}\right)-\frac{1}{2} \mathbb{I}\right) \mathcal{R}^{T}\left(p_{N}, q_{N}^{(r)}\right), \\
& \chi_{\left(p, q^{(r)}, p, q^{(s)}\right)}^{\mathcal{S}} \text { as in (8) at the top of the next page, }
\end{aligned}
$$

where $\mathbf{b}_{k}$ is a generic column of $\mathbf{B}_{k}, \mathbf{f}(m)$ is the $m$-th column of $\mathbf{F}_{2 M}$, $\odot$ represents the element-wise product.

Note that distortion expression (6) holds true for any number of users. In particular, for $K=1$, it represents the distortion of a single-user FBMC link. More specifically, for a properly tuned equalizer, we have $\operatorname{Im}\left[W_{m} H_{m}\right]=0$ and, in turn, terms $\eta_{0,0}, \eta_{0,1}$ and $\eta_{0,2}$ are identically zero. Furthermore, simple algebra can show that the remaining term $\eta_{1,1} /\left(4 M^{2}\right)$ coincides with the distortion expression given by [5, Eq. (35)]. It is worth remarking that the distortion of the single-user case decays as $M^{-2}$, as opposed to the multi-user case (6) where terms of order $O(1)$ and $O\left(M^{-1}\right)$ also appear. This means that the interaction between users generate a distortion that is independent of the frequency selectivity of the channel and, thus, cannot be reduced by increasing the number of subcarriers. It is true, though, that the proportional number of distorted out-band subcarrier will decrease, as it will be clearer later. 


$$
\begin{aligned}
& \eta_{0,0}=2 \mathbb{I m}{ }^{2}\left[W_{m} H_{k, m}\right] \mathbb{R e} \operatorname{tr}\left[\mathbf{U}^{-} \mathcal{F}_{k}(m) \chi_{(p, q, p, q)}^{\mathcal{R}-\frac{1}{2} \mathbb{I}}+\mathbf{U}^{+} \mathcal{F}_{k}(m) \chi_{(p, q, p, q)}^{\mathcal{S}}\right] \\
& \eta_{0,1}=-2 \operatorname{Im}\left[W_{m} H_{k, m}\right] \operatorname{Im}\left[W_{m} H_{k, m}^{(1)}\right] \operatorname{Im} \operatorname{tr}\left[\mathbf{U}^{+} \mathcal{F}_{k}(m) \chi_{\left(p, q, p, q^{\prime}\right)}^{\mathcal{R}-\frac{1}{2} \mathbb{R}}+\mathbf{U}^{-} \mathcal{F}_{k}(m) \chi_{\left(p, q, p, q^{\prime}\right)}^{\mathcal{S}}\right] \\
& \eta_{0,2}=-2 \mathbb{I m}\left[W_{m} H_{k, m}\right] \operatorname{Im}\left[W_{m} H_{k, m}^{(2)}\right] \operatorname{Re} \operatorname{tr}\left[\mathbf{U}^{-} \mathcal{F}_{k}(m) \chi_{\left(p, q, p, q^{\prime \prime}\right)}^{\mathcal{R}-\frac{1}{2} \mathbb{I}, \mathcal{R}}+\mathbf{U}^{+} \mathcal{F}_{k}(m) \chi_{\left(p, q, p, q^{\prime \prime}\right)}^{\mathcal{S}}\right] \\
& \eta_{1,1}=2 \operatorname{Im}^{2}\left[W_{m} H_{k, m}^{(1)}\right] \operatorname{Re} \operatorname{tr}\left[\mathbf{U}^{+} \mathcal{F}_{k}(m) \chi_{\left(p, q^{\prime}, p, q^{\prime}\right)}^{\mathcal{R}}+\mathbf{U}^{-} \mathcal{F}_{k}(m) \chi_{\left(p, q^{\prime}, p, q^{\prime}\right)}^{\mathcal{S}}\right] \\
& +2 \mathbb{R e}^{2}\left[W_{m} H_{k, m}^{(1)}\right] \mathbb{R e} \operatorname{tr}\left[\mathbf{U}^{-} \mathcal{F}_{k}(m) \chi_{\left(p, q^{\prime}, p, q^{\prime}\right)}^{\mathcal{R}}+\mathbf{U}^{+} \mathcal{F}_{k}(m) \chi_{\left(p, q^{\prime}, p, q^{\prime}\right)}^{\mathcal{S}}\right] \\
& \chi_{\left(p, q^{(r)}, p, q^{(s)}\right)}^{\mathcal{S}}=\left[\begin{array}{cc}
\mathcal{S}_{1}\left(p_{N}, q_{N}^{(r)}\right) \mathcal{S}_{1}^{T}\left(p_{N}, q_{N}^{(s)}\right) & {\left[0, \mathcal{S}_{1}\left(p_{N}, q_{N}^{(r)}\right)\right]\left[\mathcal{S}_{2}\left(p_{N}, q_{N}^{(s)}\right), 0\right]^{T}} \\
{\left[\mathcal{S}_{2}\left(p_{N}, q_{N}^{(r)}\right), 0\right]\left[0, \mathcal{S}_{1}\left(p_{N}, q_{N}^{(s)}\right)\right]^{T}} & \mathcal{S}_{2}\left(p_{N}, q_{N}^{(r)}\right) \mathcal{S}_{2}^{T}\left(p_{N}, q_{N}^{(s)}\right)
\end{array}\right]
\end{aligned}
$$

1) Interpretation: The distortion expression given by (6) and (7) can be easily evaluated with any numerical software. Nevertheless, it gives little intuition about the behavior of the distortion itself. The following remarks should be useful to grasp some more insight into the distortion generation mechanism. First of all, let us consider the classic equalizer $W_{m}=H_{k, m}^{-1}$ for $m \in \mathcal{I}_{k}$. Then, as just mentioned, coefficients $\eta_{0,0}, \eta_{0,1}$ and $\eta_{0,2}$ only contribute to the out-band distortion since they are identically null for subcarriers associated to user $k$. Besides, the terms corresponding to $\eta_{0,0}$ and $\eta_{0,1}$ in (6) decay slower than the other two. As a result, it is reasonable to expect that, for each user, out-band distortion is higher than the in-band one. Fortunately, however, this is true only for a reduced number of out-band subcarriers.

To prove the last claim, we manipulate the first term of $\eta_{0,0}$, namely

$$
\eta_{0,0}^{(1)}=2 \mathbb{I m}^{2}\left[W_{m} H_{k, m}\right] \operatorname{Retr}\left[\mathbf{U}^{-} \mathcal{F}_{k}(m) \chi_{(p, q, p, q)}^{\mathcal{R}-\frac{1}{2} \mathbb{I}}\right]
$$

and clarify its dependence on the prototype pulses and on the set $\mathcal{I}_{k}$, i.e. the set of subcarriers assigned to user $k$. The analysis of the other terms is not reported due to space constraints, but yields similar results.

In order to consider the simplest case, we fix the overlapping factor to $\kappa=1$ and we choose the same pulse at both the transmitter and the receiver sides (i.e. $q_{N}[n]=p_{N}[n]$ ). Obviously, all previous assumptions about symmetry, smoothness and reconstruction capabilities still hold true. As a result, matrix $\mathcal{R}\left(p_{N}, q_{N}\right)$, defined in (3a), takes the form $\mathcal{R}\left(p_{2 M}, p_{2 M}\right)=$ $\mathbf{r}=\left[\begin{array}{lll}p^{2}[1] & \cdots & p^{2}[2 M]\end{array}\right]^{T}$. Denote by $\boldsymbol{\rho}=\left[\rho_{1} \cdots \rho_{2 M}\right]$ the DFT of $\mathbf{r}$, that is $\boldsymbol{\rho}=\mathbf{F}_{2 M}^{H} \mathbf{r}$. Then, one can show that

$$
\eta_{0,0}^{(1)}=4 \operatorname{Im}^{2}\left[W_{m} H_{k, m}\right] \sum_{n=1}^{M}\left|\rho_{2 n}\right|^{2} \beta_{k, 2 n-m+1}
$$

where $\beta_{k, n}=\mathbb{E}\left[b_{k, n}^{2}\right] /(2 M)$. We will now show that $\eta_{0,0}^{(1)}$ is zero for most $m \notin \mathcal{I}_{k}$. Let us assume that $\mathcal{I}_{k}=$ $\left\{m_{\min }, \ldots, m_{\max }\right\}$, with $1 \leq m_{\min }<m_{\max } \leq 2 M$. Then, $\beta_{k, n}>0$ only if $m_{\min } \leq n \leq m_{\max }$ and null otherwise. On the other hand, typical prototype pulses are low-pass filters and $\rho_{n} \neq 0$ only for $1 \leq n \leq m_{0}$ and for $2 M-m_{0}+2 \leq n \leq 2 M$ $\left(\rho_{2 M-n+2}=\rho_{n}\right.$ since $p_{N}[n]$ is real $)$, normally with $m_{0}$ taking integer values close to $2 \kappa$ (see, e.g., [8]). These two facts imply that most of the terms of the last sum in (9) are zero. More specifically, a careful inspection on the indices shows that $\eta_{0,0}^{(1)}$ is not zero only if ${ }^{1} m_{\min }-m_{0}+1 \leq m \leq m_{\max }+m_{0}-1$.

Similar results can be found for all the other terms of (6), thus showing that the distortion generated by each user leaks only into the first $m_{0}-1$ subcarriers on both sides of its assigned band. It is worth recalling that the number $m_{0}$ is characteristic of the employed prototype pulse.

\section{NumeriCAl ANALYSIS}

In order to evaluate the degree of accuracy of the above results, we compare them with some simulation experiments obtained for a reasonable value of $2 M$. We consider a system with $2 M=128$ subcarriers. The bandwidth assigned to each subcarrier is $15 \mathrm{kHz}$, which corresponds to a sampling frequency of $1.920 \mathrm{MHz}$ (thus compliant with the LTE standard). The prototype pulse proposed by the PHYDYAS EU-funded project [7], with an overlapping factor $\kappa=4$ (see Fig. 2), is employed both at the transmitters (users' side) and at the receiver (base station side). The channels between each user and the base station are considered independent to one another and modeled according to either the ITU Extended Pedestrian A (EPA) model or the ITU Extended Vehicular A (EVA) model [9]. For subcarrier $m$ assigned to user $k$ (i.e. $m \in \mathcal{I}_{k}$ ), the equalizer tap $W_{m}$ is set to the inverse of the channel coefficient $H_{k, m}$. The number of users is set to two and the subcarriers are equally split among them (subcarriers 1-64 to user 1 and subcarriers $65-128$ to user 2). The transmitted symbols are modulated according to a 4QAM constellation with power $P_{s}$.

The selected pulse is characterized by the Fourier coefficients reported in Table I (with a real pulse we only need to specify the first half of the coefficients). Since the number of nonzero coefficients is as low as four, from the considerations above we expect that the distortion generated by each user leaks into very few out-band subcarriers. This is indeed what

\footnotetext{
${ }^{1}$ Index algebra is modulo $2 M$.
} 
TABLE I

Pulse FOURIER COEFFICIENTS
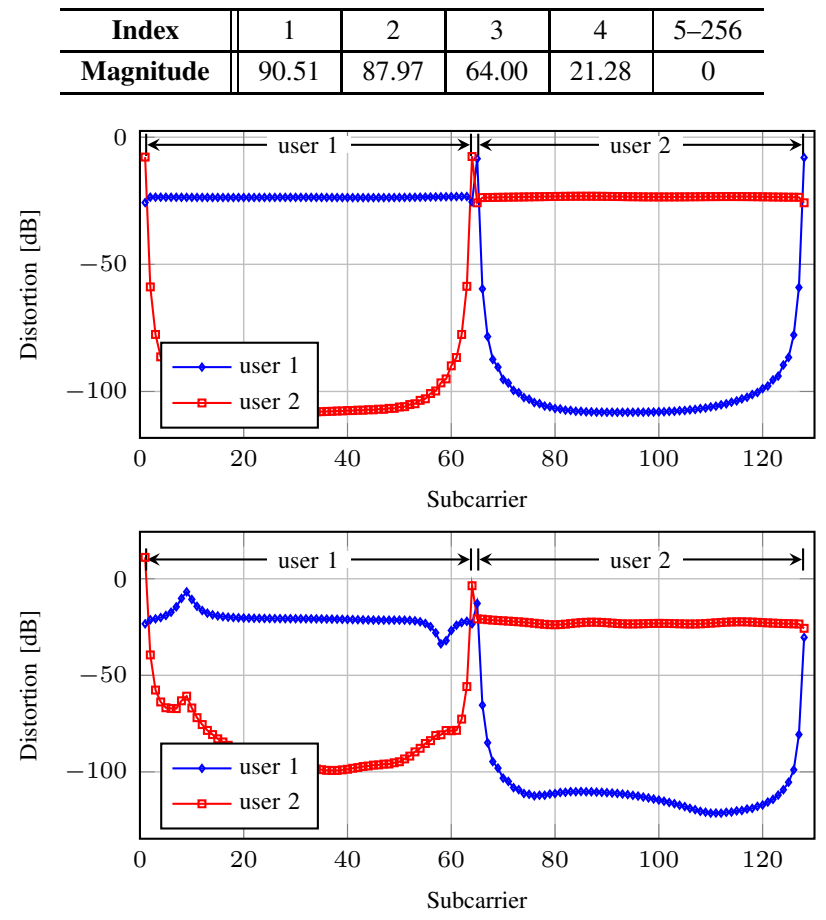

Fig. 3. Normalized distortion power for user 1. Example of EPA channel model (top) and of EVA channel model (bottom).

can be evinced from Fig. 3, where the function $P_{e, k}(m) / P_{s}$ [according to (6)] is reported for both users and both channel models. Fortunately, not only the number of affected outband subcarriers is low, but also the distortion decreases very fast and takes a noticeable value only in the first out-band subcarrier.

From Fig. 3 one also reads that the value of the in-band distortion is around $-23 \mathrm{~dB}$ for both channels. Note that this is the upperbound value when measuring the performance of the system in terms of Signal-to-Noise-plus-Distortion Ratio (SNDR)

$$
\operatorname{SNDR}(m)=\frac{P_{s}}{P_{e}(m)+P_{w}(m)}
$$

as we increase the signal to noise ratio $P_{s} / \sigma^{2}$, with $P_{w}(m)=$ $\sigma^{2}\left|W_{m}\right|^{2} M^{-1} \sum_{n=1}^{N} q_{N}^{2}[n]$ the noise power at the filterbank output. This fact can be observed in Fig. 4: the SNDR improves by a mere $6 \mathrm{~dB}$ when increasing $P_{s} / \sigma^{2}$ from $20 \mathrm{~dB}$ to $40 \mathrm{~dB}$. In particular, for $P_{s} / \sigma^{2}=40 \mathrm{~dB}$, the SNDR is approximately $23 \mathrm{~dB}$, which is in accord with Fig. 3 . Together with the theoretic curves (lines), Fig. 4 also reports the empirical ones (markers, averaged over 2000 multicarrier symbols), showing an excellent match.

\section{CONCLusions}

In this paper we have investigated the performance of a frequency division multiple-access scheme based on OQAM/FBMC. The signal distortion, which is due to suboptimal synchronization and equalization under strong frequency
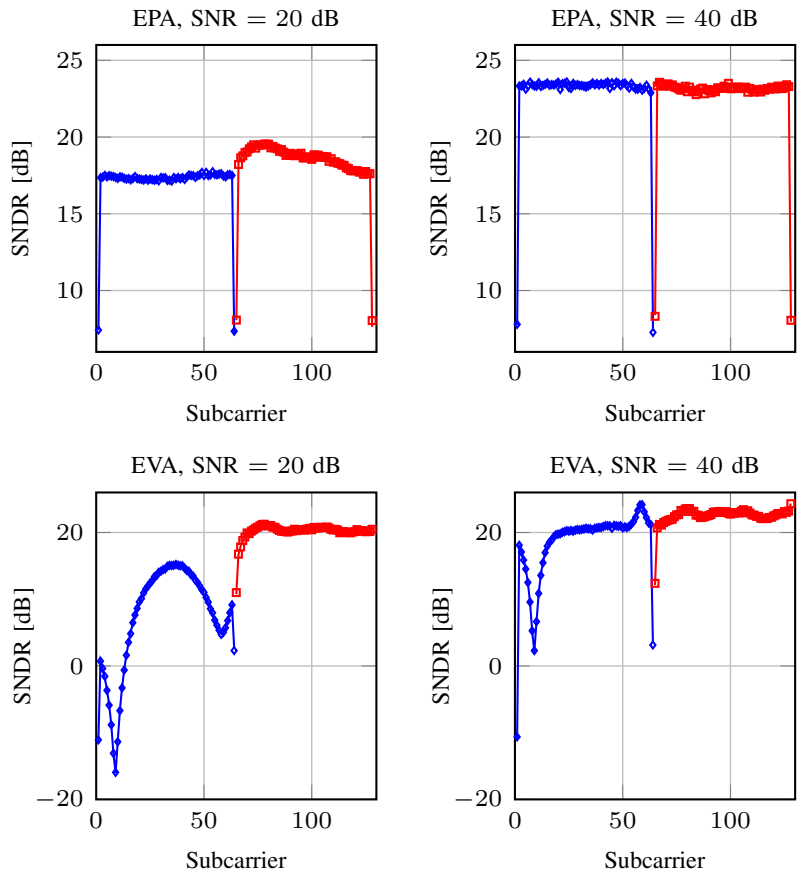

Fig. 4. Theoretic (line) and empirical (markers) SNDR for the two-user case. The ratio $P_{s} / \sigma^{2}$ is set to $20 \mathrm{~dB}$ (left) and $40 \mathrm{~dB}$ (right). Reported examples correspond to EPA channel model (top) and EVA channel model (bottom).

selectivity and is inherent to the FBMC scheme even with perfect prototype pulses, has been formulated as a sum of user contributions. More specifically, it has been shown how each user contributes to the distortion in its assigned subcarriers and in few subcarriers on the sides of its spectrum. Since the out-band distortion depends on the prototype pulses and on the channel, the distortion expression given here can be used as a start point to optimize the pulses and the equalizer.

\section{REFERENCES}

[1] H. Li and H. Liu, "An analysis of uplink OFDMA optimality," IEEE Trans. Wireless Commun., vol. 6, no. 8, pp. 2972-2983, Aug. 2007.

[2] M. Morelli, C.-C. J. Kuo, and M.-O. Pun, "Synchronization techniques for orthogonal frequency division multiple access (OFDMA): A tutorial review," Proc. IEEE, vol. 95, no. 7, pp. 1394-1427, Jul. 2007.

[3] P. Siohan, C. Siclet, and N. Lacaille, "Analysis design of OFDMA/OQAM systems based on filterbank theory," IEEE Trans. Signal Process., vol. 50, no. 5, pp. 1170-1183, May 2002.

[4] H. Saeedi-Sourck, Y. Wu, J. W. M. Bergmans, S. Sadri, and B. FarhangBoroujeny, "Complexity and performance comparison of filter bank multicarrier and OFDM in uplink of multicarrier multiple access networks," IEEE Trans. Signal Process., vol. 59, no. 4, pp. 1907-1912, Apr. 2011.

[5] X. Mestre, M. Majoral, and S. Pfletschinger, "An asymptotic approach to parallel equalization of filter bank based multicarrier signals," IEEE Trans. Signal Process., vol. 61, no. 14, pp. 3592-3606, Jul. 2013.

[6] B. Farhang-Boroujeny, "Filter bank spectrum sensing for cognitive radios," IEEE Trans. Signal Process., vol. 56, no. 5, pp. 1801-1811, May 2008.

[7] A. Viholainen et al., "Prototype filter and structure optimization," Project PHYDYAS ICT-211887, Deliverable D5.1, Jan. 2009.

[8] S. Mirabbasi and K. Martin, "Overlapped complex-modulated transmultiplexer filters with simplified design and superior stopbands," IEEE Trans. Circuits Syst. II, vol. 50, no. 8, pp. 456-469, Aug. 2003.

[9] 3rd Generation Partnership Project, "Evolved universal terrestrial radio access (e-UTRA); user equipment (UE) radio transmission and reception," ETSI, Tech. Rep. TR36.803 v1.1.0 release 8, 2008. 